\title{
The Working Surface of a Powerful Radio Source: Global MkIII Observations of the Southern Lobe of 3C205.
}

\author{
Colin J. Lonsdale \\ Haystack Observatory, Westford MA 01886 \\ Peter D. Barthel \\ California Institute of Technology, Pasadena, CA
}

The radio source $3 \mathrm{C} 205$ is identified with a quasar of redshift 1.53 , and is, at first glance, a standard, powerful double radio source with hotspots. Upon closer examination (MERLIN, VLA A-array), the southern lobe is seen to consist of two distinct hotspots plus some more diffuse emission, some 50 kiloparsecs from the quasar $\left(\mathrm{H}_{\circ} \sim 75\right)$. The arcsecond-scale morphology and polarization structure of this hotspot complex strongly suggests that the larger and weaker off-axis secondary hotspot has been formed by outflow of material from the more compact primary hotspot (Lonsdale and Barthel 1984,6). This interpretation implies a collision between the presumed energy supply beam and something capable of deflecting the flow energy towards the secondary. Because the flow is so energetic, this obstacle would have to be both massive and dense. The main constraint on the density of the obstacle comes from measurements of the internal energy density of the compact primary hotspot coupled with model-dependent estimates for the maximum advance velocity of this feature, thus yielding a balance with the ram-pressure exerted by the obstacle medium.

Previous VLBI measurements of the primary hotspot were presented in Lonsdale and Barthel (1984), and consisted of a 4-station EVN Mark II $18 \mathrm{~cm}$ hybrid map at 30 milliarcsecond resolution with approximately 10:1 dynamic range. The structure thus revealed consisted of a ridge of emission some 50 milliarcseconds in length and less than about 15 milliarcseconds wide, oriented roughly along the line joining the primary and secondary hotspots. There was also evidence for curvature of the ridgeline at one end towards the quasar, which gave the subjective impression of continuity from the quasar, through the primary hotspot to the secondary hotspot.

In order to probe the unresolved emission further, we conducted a global 7station Mark III experiment at $18 \mathrm{~cm}$, wherein the longest baselines had a fringe spacing of 4 or 5 milliarcseconds. Fringes were detected on all the short baselines (inter-Europe, Haystack-Greenbank, OVRO-VLA), and intermittently on the most sensitive longer baselines (Greenbank-VLA and all transatlantic baselines except those involving Westerbork, Haystack and OVRO). A map made from the shorter baselines is shown, and has a restoring CLEAN beam of FWHM 15 milliarcseconds. On the longer baselines, the source is detected clearly over only 
a small range of hour angles, and during that time the visibility rises steeply to between 5 and $20 \mathrm{mJy}$. This behaviour has been fitted to a narrow ridge of length 10 milliarcseconds and axial ratio 5 , in the same position angle as the main ridge in the map. What this amounts to is a faint but extremely narrow (probably still unresolved) linear feature pointing towards the secondary hotspot.

The preliminary results presented here were obtained without the benefit of sophisticated fringe-fitting and mapping techniques. We anticipate that the use of a global fringe-fitting algorithm and careful data editing will allow detection of fringes in a larger fraction of the long baseline data than we can presently claim, and that we will be able to produce a reliable hybrid map of this feature at 4 milliarcsecond resolution. Much of the data is clearly close to the detection threshold, as the closure phases appear to be non-random for substantial stretches.

The implications of these results for the interpretation of 3C205 include the need to postulate extremely dense clouds of matter more than 50 kiloparsecs from the parent galaxy. The nature of the deflection interaction should be constrained by the presence of the compact ridge, which some may choose to interpret as the long-hypothesized "working surface" where the jet impinges on the external medium (in this case a dense cloud). Finally, the detection of milliarcsecond structure in the outer lobe of a quasar raises the possibility of directly measuring the relative motion between the hotspot and core, one of the more important unknown quantities in radio source physics.

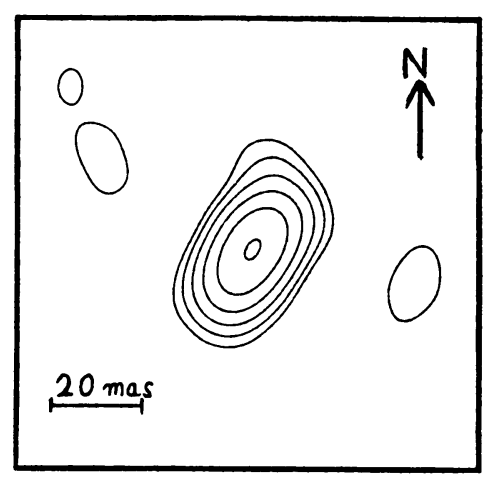

Fig. 1. MkIII VLBI map of 3C205 southern hotspot. The peak is about 50 $\mathrm{mJy} / \mathrm{beam}$, and the resolution is about 15 milliarcseconds. The bottom contour is $3 \%$ of the peak. This is a preliminary map utilizing only the shorter baselines (see text).

\section{REFERENCES}

Lonsdale C.J. and Barthel P.D., 1984, Astron. Astrophys. 135, 45.

Lonsdale C.J. and Barthel P.D., 1986, Astron J. 92, 1. 